\title{
Persistent Dystrophin Protein Restoration 90 Days after a Course of Intraperitoneally Administered Naked 2'OMePS AON and ZM2 NP-AON Complexes in mdx Mice
}

\author{
Elena Bassi, ${ }^{1}$ Sofia Falzarano, ${ }^{1}$ Marina Fabris, ${ }^{1}$ Francesca Gualandi, ${ }^{1}$ \\ Luciano Merlini, ${ }^{2}$ Gaetano Vattemi, ${ }^{3}$ Daniela Perrone, ${ }^{4}$ Elena Marchesi, ${ }^{4}$ \\ Patrizia Sabatelli, ${ }^{5}$ Katia Sparnacci, ${ }^{6}$ Michele Laus, ${ }^{6}$ Paolo Bonaldo, ${ }^{7}$ \\ Paola Rimessi, ${ }^{1}$ Paola Braghetta, ${ }^{7}$ and Alessandra Ferlini ${ }^{1}$ \\ ${ }^{1}$ Department of Experimental and Diagnostic Medicine, Section of Medical Genetics, University of Ferrara, Via Fossato di Mortara 74, \\ 44100 Ferrara, Italy \\ ${ }^{2}$ Department of Physical and Rehabilitation Medicine, Rizzoli-Sicily IOR, 90011 Bagheria, Italy \\ ${ }^{3}$ Department of Neurological Sciences and Vision, Section of Clinical Neurology, University of Verona, 37129 Verona, Italy \\ ${ }^{4}$ Department of Biology and Evolution, University of Ferrara, 44121 Ferrara, Italy \\ ${ }^{5}$ IGM-CNR, Unit of Bologna, c/o IOR, 40136 Bologna, Italy \\ ${ }^{6}$ Department of Environmental and Life Sciences INSTM, University of Eastern Piedmont, 15121 Alessandria, Italy \\ ${ }^{7}$ Department of Histology, Microbiology, and Medical Biotechnology, University of Padua, 35121 Padua, Italy
}

Correspondence should be addressed to Alessandra Ferlini, fla@unife.it

Received 21 March 2012; Revised 25 May 2012; Accepted 14 June 2012

Academic Editor: Steve Winder

Copyright ( $) 2012$ Elena Bassi et al. This is an open access article distributed under the Creative Commons Attribution License, which permits unrestricted use, distribution, and reproduction in any medium, provided the original work is properly cited.

\begin{abstract}
In Duchenne muscular dystrophy, the exon-skipping approach has obtained proof of concept in animal models, myogenic cell cultures, and following local and systemic administration in Duchenne patients. Indeed, we have previously demonstrated that low doses $(7.5 \mathrm{mg} / \mathrm{Kg} /$ week) of 2'-O-methyl-phosphorothioate antisense oligoribonucleotides (AONs) adsorbed onto ZM2 nanoparticles provoke widespread dystrophin restoration 7 days after intraperitoneal treatment in mdx mice. In this study, we went on to test whether this dystrophin restoration was still measurable 90 days from the end of the same treatment. Interestingly, we found that both western blot and immunohistochemical analysis (up to 7\% positive fibres) were still able to detect dystrophin protein in the skeletal muscles of ZM2-AON-treated mice at this time, and the level of exon-23 skipping could still be assessed by RT real-time PCR (up to $10 \%$ of skipping percentage). In contrast, the protein was undetectable by western blot analysis in the skeletal muscles of mdx mice treated with an identical dose of naked AON, and the percentage of dystrophin-positive fibres and exon-23 skipping were reminiscent of those of untreated mdx mice. Our data therefore demonstrate the long-term residual efficacy of this systemic low-dose treatment and confirm the protective effect nanoparticles exert on AON molecules.
\end{abstract}

\section{Introduction}

Duchenne muscular dystrophy (DMD) is an inherited Xlinked degenerative muscle disorder mainly caused by framedisrupting mutations following large rearrangements in the dystrophin gene [1]. DMD boys are affected by severe skeletal muscle wasting and cardiomyopathy. However, therapeutic approaches for this debilitating disease are now a realistic hope.
Antisense-oligoribonucleotide (AON)-mediated exon skipping [2-4] has indeed already entered into clinical trials in humans. These trials are concentrating on local injection $[5,6]$ and systemic administration $[7,8]$ of two different chemicals 2'-O-methyl-phosphorothioate (2'OMePS) and phosphorodiamidate morpholino oligomer (PMO), both administered naked. Regarding the dose regimens tested, the local injection studies used $0.8 \mathrm{mg}$ of PRO051/GSK2402968 AON (2'OMePS backbone) and both/either $0.09 \mathrm{mg}$ and/or 
$0.9 \mathrm{mg}$ of AVI-4658 AON (PMO backbone), inducing exon51 skipping in all cases $[5,6]$. In phase I/II systemic clinical trials, AVI-4658 and PRO051/GSK2402968 have been administered by intravenous (i.v.) and subcutaneous (s.c.) injection, using incremental doses from 0.5 to $20.0 \mathrm{mg} / \mathrm{Kg}$ and from 0.5 to $6.0 \mathrm{mg} / \mathrm{Kg}$, respectively [7, 8]. Although for the most part studies are ongoing, they have already revealed the absence of severe adverse effects, at least at the doses tested, and confirmed the therapeutic potential of specific exon skipping to induce dystrophin restoration in DMD patients.

Preclinical studies on the mdx mouse (the most frequently studied animal model of dystrophy) are also underway, with a view to identifying the most appropriate and safe delivery system for the AON molecules, irrespective of their chemical formulation. The aims of these studies are to (i) ensure more efficient muscle targeting, and (ii) define the optimal effective therapeutic AON dose that will enable the chronic life-long treatment required by DMD patients.

Rather inconveniently, the different chemical properties of the two AON backbones preclude the use of a universal carrier for efficient delivery. Nevertheless, recent studies have described the use of PEG-PEI copolymers and nonionic polymersomes as efficient carriers for local delivery of $2^{\prime} \mathrm{OMePS}$ AONs in mdx mice $[9,10]$. Furthermore, a new formulation of PEG-PEI copolymer associated with functionalized derivatives containing either the cell-penetrating peptide TAT, adsorbed colloidal gold, or both, have also yielded promising results [11]. Grounds for optimism have also been provided by an approach exploiting a set of lipid nanoparticles with different compositions of cationic lipids and polyethylene glycol (PEG) when tested for their ability to deliver a luciferase siRNA to the liver via systemic administration in mice $[12,13]$. Moreover, chitosan-coated nanoparticles have been used in mice as carriers for the in vivo delivery of active siRNA to papillary thyroid carcinoma by systemic administration [14]. Unfortunately, however, activation of the complement system by conversion of $\mathrm{C} 3$ into C3b in serum incubated with chitosan-coated nanoparticles is known to cause significant side effects [15]. Hopes are therefore pinned on a clinical trial involving the systemic administration of siRNA using targeted nanoparticles as a delivery system in patients with solid cancers that is currently underway [16].

Regarding our efforts in the field, we have previously demonstrated that nonviral biocompatible nanoparticles (NPs) (named T1 and ZM2) bind and deliver 2'OMePS M23D AON in mdx mice by systemic intraperitoneal (I.P.) injections. These complexes showed a body-wide distribution and efficiently induced dystrophin restoration in the skeletal muscles of the quadriceps, gastrocnemius and diaphragm, the arrector pili smooth muscle, and the cardiac muscle of the heart, as assessed by a routine cohort of biochemical outcome measures (skipping quantification, immunostaining, and positive fibres counting, as well as western blotting). Our results also suggest that these nanoparticles could afford protection to antisense RNA molecules [17, 18]. Moreover, the use of ZM2 nanoparticles in particular has allowed us to employ very low doses of
M23D AON (7.5 mg/Kg/week, $52.5 \mathrm{mg} / \mathrm{Kg}$ in total), and to observe the efficacy of this systemic treatment at 1 week after the last injection [18].

In this further study, we tested whether the protective effect of ZM2 nanoparticles on AON molecules noted was still measurable at 3 months from the end of the same low dosage treatment $(52.5 \mathrm{mg} \mathrm{AON} / \mathrm{Kg}$ in total) and whether the restored dystrophin protein was detectable by the same outcome measures employed in our previous work [18]. Encouragingly, we discovered the long-term persistence of reframed dystrophin protein expression in the skeletal muscles of mdx mice following treatment with the same low dosage of M23D AON, corresponding to $1 / 20$ of the effective I.P. dosage described for naked AONs [19]. We show that the effect of ZM2-AON on dystrophin expression persists up to 3 months (western blot positive, skipping percentage up to $10 \%$, and immunofluorescence positive fibres up to $7 \%$ ) from the suspension of treatment, contrasting with results yielded by an identical naked AON dose regimen (western blot negative, skipping percentage, and immunofluorescence positive fibres reminiscent of untreated $\mathrm{mdx}$ mice).

\section{Materials and Methods}

2.1. Animals. All experiments were performed on male $\mathrm{mdx}$ mice $(\mathrm{C} 57 \mathrm{BL} / 10 \mathrm{ScSn}-\mathrm{Dmdmdx} / \mathrm{J})$ and age-matched wildtype (WT) male mice (C57BL/10SnJ). All procedures were approved by the Animal Experimentation Ethics Committee. Mice were purchased from the Jackson Laboratory (Bar Harbor, ME) and housed in temperature-controlled rooms $\left(22^{\circ} \mathrm{C}\right)$ at a humidity level of $50 \%$ and a $12: 12$ hour lightdark cycle.

2.2. ZM2-M23D AON-Loading Experiments. ZM2s are cationic core-shell nanoparticles made up of a PMMA core surrounded by a random copolymer shell consisting of units derived from $\mathrm{N}$-isopropyl acrylamide+ (NIPAM) and reactive methacrylate-bearing cationic groups [18]. M23D AON, which contains a $2^{\prime}$-O-methyl RNA phosphorothioate backbone, was synthesized as previously described [17]. Loading experiments showed that ZM2 nanoparticles $(1 \mathrm{mg} / \mathrm{mL})$ adsorbed 2'OMePS M23D oligoribonucleotide onto their surface in the concentration range $10-100 \mu \mathrm{g} / \mathrm{mL}$. M23D adsorption onto ZM2 nanoparticles is a highly reproducible process with a loading value of $90 \mu \mathrm{g} / \mathrm{mg}$ [18].

\subsection{I.P. Injections of ZM2-M23D AON Complexes or Naked} M23D AON in mdx Mice. Mdx male mice (6 weeks of age) were I.P. injected with either $200 \mu \mathrm{L}$ of ZM2-M23D complex containing $225 \mu \mathrm{g}$ of M23D AON and $2.5 \mathrm{mg}$ of ZM2 nanoparticles dissolved in sterile, unpreserved saline solution ( $0.9 \%$ sodium chloride), or $200 \mu \mathrm{L}$ containing $225 \mu \mathrm{g}$ of M23D dissolved in unpreserved saline solution (naked AON) and monitored according to approved NIH and University guidelines. A summary of the injection schedule is shown in Table 1. Four additional mdx mice were injected with $200 \mu \mathrm{L}$ containing $2.5 \mathrm{mg}$ of ZM2 NPs dissolved in sterile unpreserved saline solution $(0.9 \%$ sodium chloride). The 
total amount of M23D AON received by each animal was $7.5 \mathrm{mg} / \mathrm{Kg} /$ week, $52.5 \mathrm{mg} / \mathrm{Kg}$ in total. One group of $\mathrm{mdx}$ mice $(n=4)$ received seven injections of ZM2-M23D complex once a week for seven weeks, the second group $(n=4)$ received seven injections of M23D-naked AON, and the third $(n=4)$ received seven injections of ZM2 NPs at the same times. Four noninjected (not treated) age-matched mdx mice were used as controls.

2.4. RNA Studies. Total RNA was extracted from frozen sections of muscle biopsies using TRIzol (Invitrogen, Milan, Italy), and reverse-transcribed into cDNA using the HighCapacity cDNA Reverse Transcription kit (Applied Biosystems, Frankfurt, Germany).

Exon-specific real time assays (ESRAs) [20] were used on exons 8,23 , and 25 to quantify the percentage of exon-23 skipping in treated, with respect to untreated mice $(\Delta \Delta \mathrm{Ct}$ method), using $\beta$-actin as endogenous control, as previously described [17].

2.5. Immunofluorescence Analysis. At 12 weeks after the last injection, all $\mathrm{mdx}$ mice were killed, and their diaphragm, quadriceps and cardiac muscles isolated, blotted dry, trimmed of external tendon, snap-frozen in liquid $\mathrm{N}_{2}$-cooled isopentane, and stored at $-80^{\circ} \mathrm{C}$ until further processing. Seven-micrometer-thick frozen transverse sections were cut from at least two-thirds of the length of the heart, diaphragm and quadriceps muscles, and for each muscle at least 5 slices were cut at $150 \mu \mathrm{m}$ intervals.

To analyse dystrophin restoration, freshly cut muscle sections were labelled with a polyclonal antidystrophin antibody (H-300, diluted 1:100; Santa Cruz Biotechnology, Santa Cruz, CA) and revealed with anti-rabbit Cy3-conjugated secondary antibody (Jackson Immunoresearch, Suffolk, UK). For the semiquantitative analysis of dystrophin, all samples were double-labelled with a rat monoclonal antibody for laminin-2 (a2-chain, 4H8-2, diluted 1:500; Alexis Biochemical, Farmingdale, NY, USA), followed by anti-rat Cy2conjugated secondary antibody (Jackson Immunoresearch, Suffolk, UK). The percentage of fibres with a dystrophin labelling that covers at least $80 \%$ of the sarcolemma perimeter was then calculated in treated (ZM2-AON and naked AON) compared to untreated (NT) mdx mice [17]. At least 1000 muscle fibres obtained from five different levels of tissue blocks for each sample were studied for statistical evaluation. All images were observed with a Nikon Eclipse 80i fluorescence microscope (Nikon Instruments, Florence, Italy) connected to at a high-resolution CCD camera (Nikon Instruments, Florence, Italy) at 20x magnification. For statistical analysis, all the data were analysed by means of Student's $t$-test.

2.6. Tissue Morphology Analysis. Sections obtained from several blocks of kidney of mdx mice treated with ZM2-AON and untreated $\mathrm{mdx}$ (control) were stained with hematoxylin and eosin (H\&E) (Sigma-Aldrich, Milan, Italy) and anticollagen I antibody (diluted 1:80, Abcam, Cambridge, UK), and observed at 10x and 20x with a Nikon Eclipse $80 \mathrm{i}$ microscope. Images were recorded with a Nikon digital camera.

2.7. Western Blot Analysis. Western blot analysis was performed as previously described [17]. Briefly, twentymicrometer-thick frozen muscle sections were homogenized with a lysis buffer $(7 \mathrm{~mol} / \mathrm{L}$ urea, $2 \mathrm{~mol} / \mathrm{L}$ thiourea, $1 \%$ amidosulfobetaine-14, and $0.3 \%$ dithioerythritol). Aliquots of proteins from wild type mice $(15 \mu \mathrm{g})$ and from the muscles of treated or untreated $\mathrm{mdx}$ mice $(150 \mu \mathrm{g})$ were loaded onto a $6 \%$ sodium dodecyl sulphate-polyacrylamide gel and separated by electrophoresis. Samples were transferred to a nitrocellulose membrane at $75 \mathrm{~V}$, and the membrane was incubated overnight at $4^{\circ} \mathrm{C}$ with the specific antibody DYS2 (NovoCastra, Newcastle, UK). To quantify the restoration of dystrophin protein in treated versus wild-type mice, a densitometric analysis of autoradiographic bands was performed with a Bio-Rad Densitometer GS 700 (Bio-Rad, Milan, Italy), followed by normalization with the quantity of total protein loaded onto the gels.

\section{Results}

3.1. I.P. Injections of ZM2-AON Complexes and Naked AON in $\mathrm{mdx}$ Mice. $12 \mathrm{mdx}$ male mice were treated (from 6 weeks of age) via intraperitoneal injections once a week for 7 weeks: 4 mice received the ZM2-AON complex $(7.5 \mathrm{mg} / \mathrm{Kg} /$ week of AON), 4 mice received naked AON $(7.5 \mathrm{mg} / \mathrm{Kg} /$ week of $\mathrm{AON}$ ), and 4 mice received the ZM2 NPs. Controls were 4 age-matched mdx mice that did not receive any treatment. All animals were sacrificed 12 weeks after the last injection (at 6 months of age). Table 1 summarizes the treatment and sacrifice of the mice analysed in the present study.

Intraperitoneal injection was adopted as the best administration route after preliminary studies showed that i.v. injections of nanoparticles via caudal vein induced venous lesions and subsequent necrotic areas in the tail in a significant number of mice, probably due to obstructing nanoparticle aggregates; furthermore, subcutaneous injection resulted in local accumulation with no circulation of nanoparticles and no AON release (Ferlini A. and Rimessi P., unpublished observations).

\subsection{Dystrophin RNA Analysis: Evaluation of Exon-23 Skipping} in $m d x$ Mice. In order to test the efficacy of the two treatments, we conducted three different experiments to assess exon-23 skipping levels, using a Real-Time PCR exonspecific assay (ESRA) of our own devising as previously reported $[17,21]$. Skipping percentages were calculated in treated with respect to untreated mice.

Figure 1 summarizes the results obtained in the quadriceps, diaphragm and heart. Small percentages of exon-23 skipping were detected in all muscles from $\mathrm{ZM} 2-\mathrm{AON}$ - and naked AON-treated mice: $9.5 \%$ in the diaphragm, $7.8 \%$ in cardiac muscle and $6.3 \%$ in the quadriceps of mice receiving $\mathrm{ZM} 2-\mathrm{AON}$ treatment, and $6.5 \%$ in the diaphragm, $3.3 \%$ in cardiac muscle and $2.6 \%$ in the quadriceps of those treated with naked AON. Only in the diaphragm and quadriceps 
TABLE 1: Injection schedule.

\begin{tabular}{|c|c|c|c|}
\hline Group ( $n^{\circ}$ of mice) & Formulations & $\mathrm{N}^{\circ}$ of I.P. injections (1/week) & Sacrifice \\
\hline Naked AON $(n=4)$ & $\begin{array}{c}\text { M23D-AON } \\
225 \mu \mathrm{g} / \text { injection } \\
(7.5 \mathrm{mg} / \mathrm{Kg} / \text { week })\end{array}$ & 7 & 12 weeks after last injection \\
\hline $\mathrm{ZM} 2-\mathrm{AON}(n=4)$ & $\begin{array}{c}\text { ZM2 } 2.5 \mathrm{mg} \\
\text { M23D-AON } \\
225 \mu \mathrm{g} / \text { injection } \\
(7.5 \mathrm{mg} / \mathrm{Kg} / \text { week })\end{array}$ & 7 & 12 weeks after last injection \\
\hline $\mathrm{ZM} 2(n=4)$ & ZM2 $2.5 \mathrm{mg} /$ injection & 7 & 12 weeks after last injection \\
\hline Not Treated $(n=4)$ & Not Treated & Not Treated & 12 weeks after last injection \\
\hline
\end{tabular}

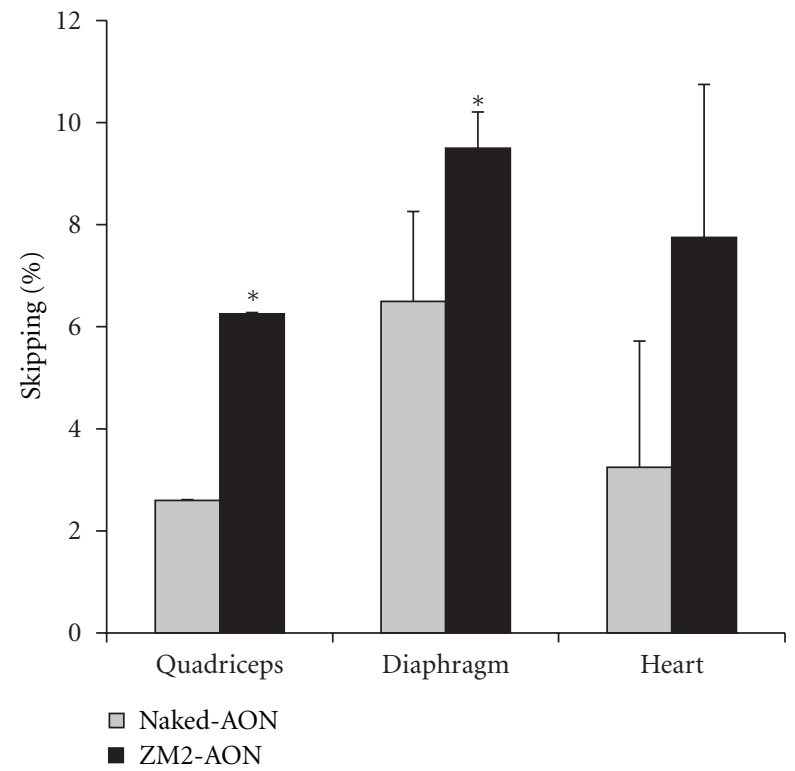

FIgURE 1: RT real time PCR exon-skipping quantification. Histograms show the percentages of exon-23-skipped transcript, calibrated on exons 8 and $25(\Delta \Delta$ Ct method) and calculated in treated (ZM2-AON and naked $\mathrm{AON}$ ) with respect to untreated $\mathrm{mdx}$ mice. The in-frame skipped product was detected in all skeletal muscles and the heart at percentages ranging from 6.3 to $9.5 \%$ in ZM2-AON-treated mice (black bars) and from 2.6 to $6.5 \%$ after treatment with naked AON (grey bars). Asterisks $(*)$ indicate statistical significance at $P<0.005$. Error bars represent mean $\pm \mathrm{SD}$.

were the differences between the treatment with or without ZM2 nanoparticles statistically significant. In fact, in the cardiac muscle from naked AON- and ZM2-AON-treated $\mathrm{mdx}$ mice, the values of exon-23 skipping (ranging from $3.3 \%$ to $7.8 \%$ ) were similar to the physiological skipping present in untreated $\mathrm{mdx}$ mice (Figure 1). In muscles from mice receiving ZM2 uncomplexed NP, the skipping percentages were similar to those measured in untreated mdx mice (data not shown).

3.3. Immunofluorescence Analysis. In order to quantify dystrophin-positive fibres and to evaluate the correct localization of dystrophin, wild-type, treated and untreated mdx muscle sections were double-labelled with anti-laminin alpha2-chain antibody, as a marker of muscle fibre basement membrane reported to be unaffected in dystrophin deficient muscle [22], and with a polyclonal antidystrophin antibody.

As shown in Figure 2(a), immunofluorescence analysis revealed the presence of dystrophin three months after the end of treatment in the quadriceps and diaphragm of both naked AON and ZM2-AON-treated mice. Dystrophin was correctly localized at the plasma membrane of muscle fibres, as confirmed by double-labelling with anti-laminin alpha2 chain antibody (not shown). Although the labelling pattern was discontinuous in most of fibres from both ZM2AON- and naked AON-treated mice, groups of fibres with continuous dystrophin labelling (greater than $80 \%$ of the fibre's perimeter) could be detected in ZM2-AON-treated samples only (Figures 2(a) and 2(b)). Semiquantitative analysis of fibres with continuous dystrophin labelling showed a significantly higher percentage after treatment with ZM2$\mathrm{AON}$ (7.2\% in the quadriceps and $4.5 \%$ in the diaphragm) with respect to naked $\mathrm{AON}$ (1.6\% in the quadriceps and $0.2 \%$ in the diaphragm), which yielded levels reminiscent of the untreated mdx mice used as control (Figure 3). The heart resulted negative (Figure 3 ).

Immunofluorescence analysis performed on muscles from ZM2 uncomplexed NP-treated mice revealed a number of dystrophin positive fibres similar to that found in control untreated mdx mice (data not shown).

3.4. Tissue Morphology Analysis. Tissue morphology analysis of mdx mice treated with ZM2-AON versus untreated mdx (control) showed no apparent tissue damage, an absence of morphological alterations, no cell architecture modifications, and an absence of inflammatory infiltrates in the kidney in either group (Figure 4).

3.5. Western Blotting. Western blot analysis, performed as previously described [17], confirmed the presence of highmolecular-weight dystrophin protein only in the quadriceps and diaphragms of ZM2-AON-treated mdx mice (Figure 5). Interestingly, no protein was detected in the muscles from mice treated with naked AON.

\section{Discussion}

These findings demonstrate the persistence of dystrophin protein in mdx mice systemically treated with $2^{\prime} \mathrm{OMePS}$ AON adsorbed onto ZM2 nanoparticles, 3 months after the 

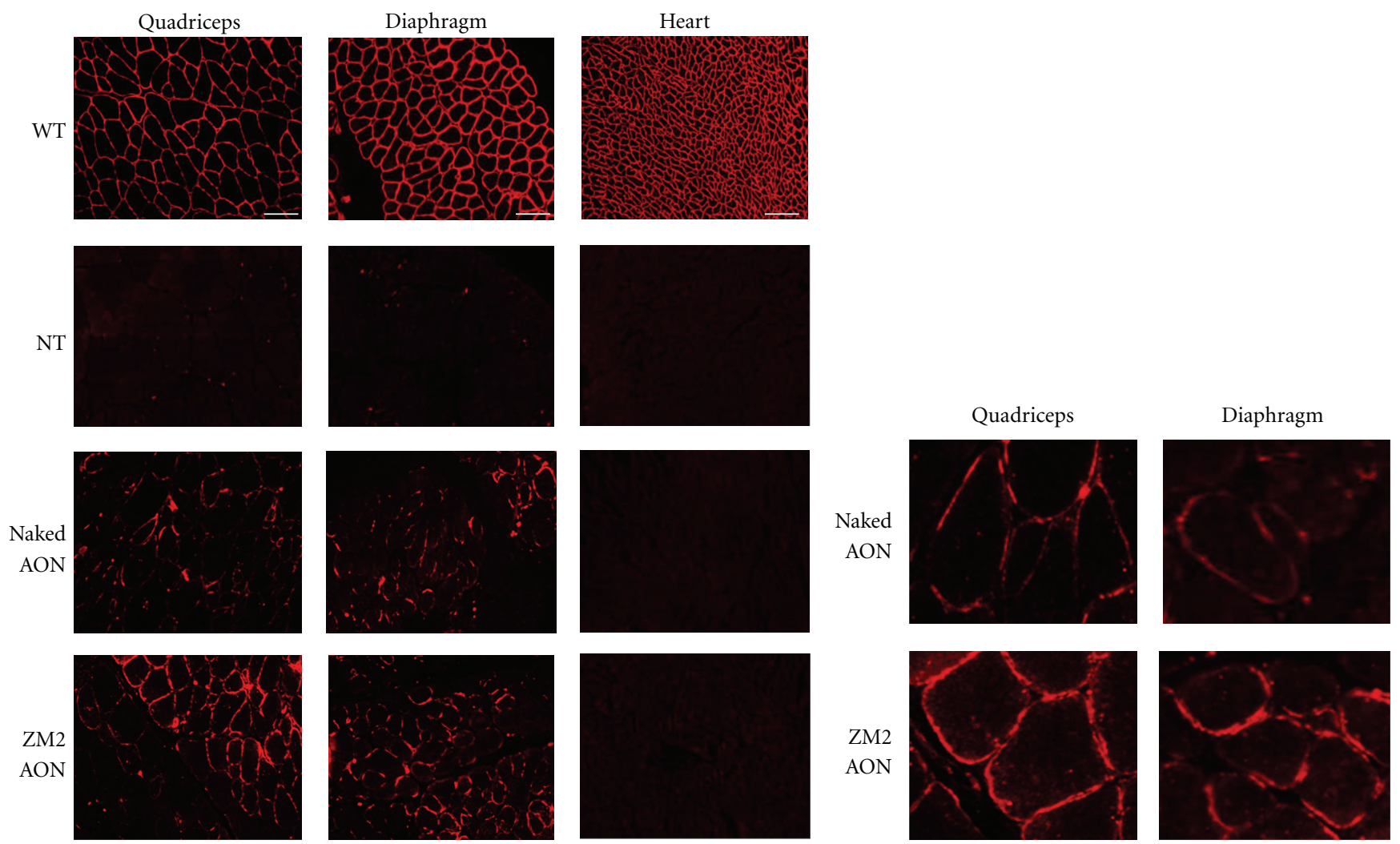

(a)

(b)

FIGURE 2: Immunofluorescence analysis. (a) Restored dystrophin is detectable and correctly expressed at the membrane of muscle fibres (quadriceps and diaphragm) from ZM2-AON-treated mdx mice. Traces are also visible in skeletal muscles from naked AON-treated mice, although the distribution at the sarcolemma is not continuous and very faint. Cardiac muscles were found to be negative for restored dystrophin. (Scale bar $=100 \mu \mathrm{m}$ in quadriceps, $50 \mu \mathrm{m}$ in diaphragms, $25 \mu \mathrm{m}$ in heart). (b) Here are represented some enlarged details of immunofluorescence images that highlight the different expression patterns of rescued dystrophin in the quadriceps and diaphragm of ZM2-AON- and naked AON-treated mdx mice. These images show both a brighter dystrophin labelling and a more continuous distribution along the fibre membranes in muscles from ZM2-AON-treated mice (NT, untreated; WT, wild type).

interruption of the treatment. At this juncture, restored dystrophin protein was still visible under immunofluorescence analysis, and shown to be correctly localized at the muscle fibre sarcolemma in the diaphragm, near to the injection site, and in the quadriceps, a distal muscle. The count of dystrophin positive fibres with continuous labelling shows a low level of positivity, although significantly higher with respect to that observed in naked $\mathrm{AON}$-treated mice. Western blotting confirmed the correct molecular weight of this almost full-length protein. This result is very encouraging considering that the low-dose treatment was suspended 3 months before the sacrifice.

To put these findings into context, although immunofluorescence analysis in naked AON-treated mice did show dystrophin-labelling in the skeletal muscles both proximal (diaphragm) and distal (quadriceps) to the site of I.P. injection, the restored protein was found to be localized irregularly at the sarcolemma (patchy). Interestingly, however, no restored protein was detected by western blot, a technique that reflects the total amount of dystrophin protein translated and present in the cells, in contrast to immunofluorescence, which mirrors only the dystrophin anchored at the sarcolemma [5].

When approaching skeletal muscles diseases with molecular therapies, it has to be considered that muscles are multinucleated syncytia and the rescue of a single-muscle fibre requires correction of multiple nuclei. In dystrophinopathy, each reverted single nucleus determines dystrophin expression in a restricted region of the fibre comprised within its nuclear domain [23]. Hence, the lower percentages of fibres with a continuous labelling pattern $(>80 \%$ of the perimeter) in mice treated with naked $\mathrm{AON}$ with respect to those treated with $\mathrm{ZM} 2-\mathrm{AON}$ could derive from a lower fraction of corrected nuclei. Although it is difficult to establish the minimal number of nuclei, it seems reasonable to suggest that a large number of nuclei have to be corrected to ensure the persistence of detectable levels of dystrophin expression [23], a theory that would explain the absence of dystrophin protein in western blot from naked AONtreated muscles. Neither treatment (naked AON or ZM2$\mathrm{AON}$ ) appeared to restore dystrophin protein in the heart, an unsurprising finding considering that cardiac muscle is the 


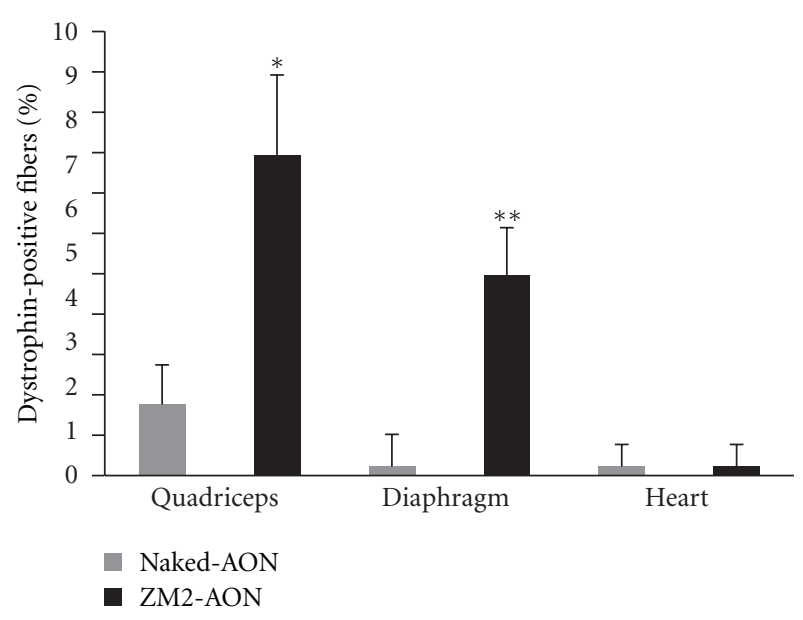

Figure 3: Dystrophin-positive fibre counting. Dystrophin-positive fibre counting was performed on fibres with a labelling that covers at least $80 \%$ of the sarcolemma perimeter. Images were recorded and processed using identical exposure parameters. The graph shows the percentage of dystrophin-positive fibres detected in the muscles and heart from ZM2-AON- (black columns) and naked AON-treated (grey columns) mdx mice, calculated with respect to untreated mice, on sections representative of the total muscle. Quadriceps and diaphragm from ZM2-AON-treated mice show significantly higher percentages of dystrophin-positive fibres (7.2\% in quadriceps and $4.5 \%$ in diaphragm) than the same muscles from mice treated with naked AON (reminiscent of untreated mdx mice). Negligible levels of dystrophin-positive fibres were detected in the heart of both treatment groups. Percentages of positive fibres in muscles from treated mice (ZM2-AON and naked AON) were calculated by subtracting the percentages detected in untreated $\mathrm{mdx}$ mice (up to $3 \%$ ), considered as baseline (\% positive fibres in treated mice- $\%$ positive fibres in untreated mice). The significance was calculated with respect to naked AON-treated mice (error bars represent mean \pm S.E.M., $n=10, t$-test, and $\left.{ }^{*} P<0.05,{ }^{* *} P<0.001\right)$.

more difficult disease target to be reached, at least when using inefficient delivery systems or low doses of AONs [19, 24, 25]. Indeed the use of peptide-conjugated PMO molecules, in particular CPP-PMO and Pip5e-PMO, have been shown as very efficient at dealing with dystrophin restoration in the heart, due to the presence of stretches of arginines and a central hydrophobic core, which enhanced nuclear PMO delivery in cardiomyocytes [26-28].

\section{Conclusions}

In conclusion, this is the first study on phosphorothioate oligoribonucleotides, both naked and loaded onto NPs, documenting a long-lasting effect at such a low dose $(7.5 \mathrm{mg} / \mathrm{Kg} /$ week, $52.5 \mathrm{mg} \mathrm{AON} / \mathrm{Kg}$ in total). Indeed, this corresponds to $1 / 20$ of the doses used for the $\mathrm{mdx}$ mouse in previous systemic studies [19,29].

At this point in time, although encouraging enough to warrant clinical trials, the results obtained in mice with naked $2^{\prime} \mathrm{OMePS} A O N$ seem to indicate that the doses required for a therapeutic effect might be too high for longterm treatment in humans. In fact, systemic naked AON

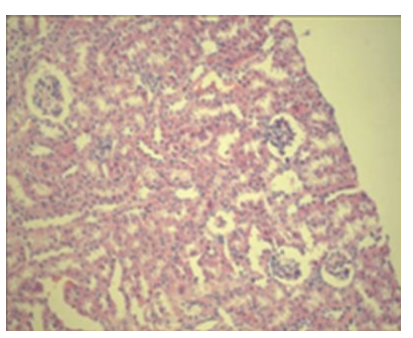

(a)

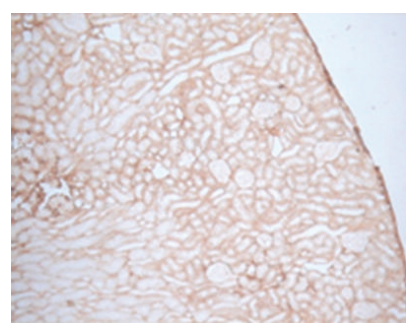

(c)

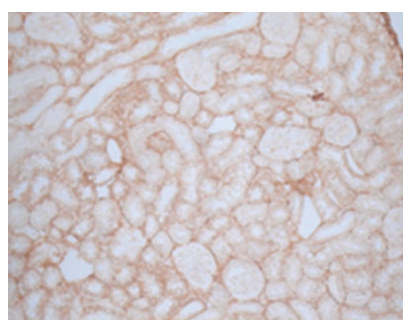

Mdx control

(e)

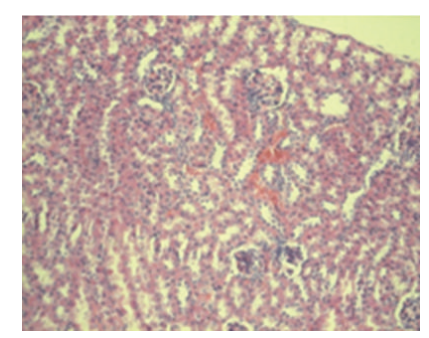

(b)

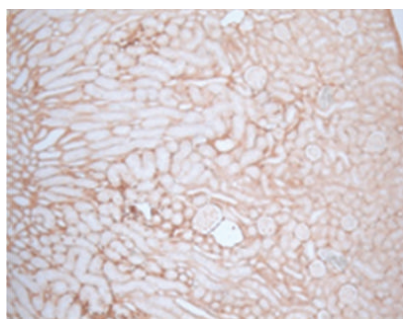

(d)

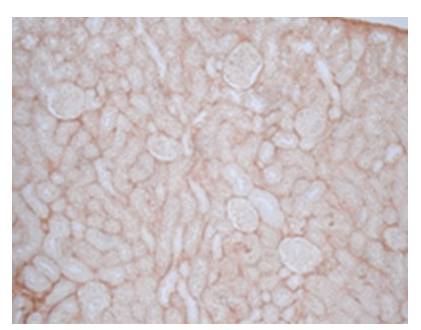

Mdx-ZM2-AON treated

(f)
FIgURE 4: Tissue morphology analysis. Histological analysis on kidneys from control and ZM2-AON-treated mdx mice. (a) and (b) Hematoxylin and Eosin staining; (c-f) immunohistochemistry with anticollagen I antibody; (a-d) 10x; (e) and (f): 20x. No morphological alterations were observed in either group of mice.

delivery in animal models only yielded satisfactory levels of dystrophin rescue in both skeletal and cardiac muscles at amounts of AON ranging from $300 \mathrm{mg} / \mathrm{Kg}$ to $1250 \mathrm{mg} / \mathrm{Kg}$ $[19,30]$. Nevertheless, better results have been reached with more moderate doses of PMO AONs, that is, roughly $600 \mathrm{mg} / \mathrm{Kg}$ [31].

Until now, the long-term persistence of restored dystrophin protein after low-dosage systemic treatment has only been achieved using naked morpholino or, more promisingly, molecules conjugated with a cell-penetrating peptide (CPP) containing a stretch of arginines [19, 32-34].

All these different AON formulations still raise safety implications, regarding their nondegradable nature (PMO, [9]), their possible toxicity (PPMO, [35]) and the renal overload $\left(2^{\prime} \mathrm{OMePS},[19,36,37]\right)$. This is especially true for long-life treatments as those required for DMD.

In this paper, we reemphasize our previous results regarding the use of ZM2 nanoparticles as a nontoxic vehicle for RNA molecules [18], and we show that the protective effect of the ZM2 remains at 3 months from the interruption of treatment. In fact, the dystrophin restoration 

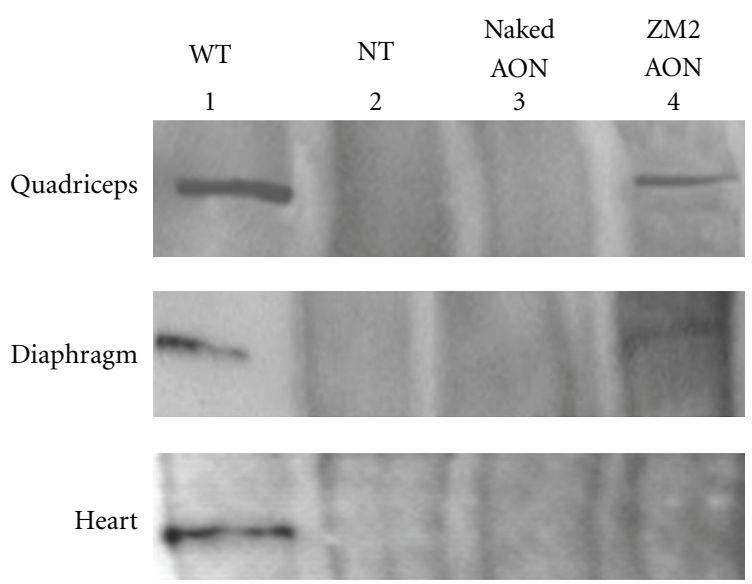

FIGURE 5: Western blot analysis. Immunoblotting for dystrophin using DYS2 antibody showed restored expression of the protein in the quadriceps and diaphragm of ZM2-AON-treated mice (lane 4), while no protein was detected in either untreated control (NT, lane 2) or naked AON-treated mdx mice (lane 3). Dystrophin protein was undetectable in the hearts of both untreated and treated mdx mice. Quadriceps, diaphragms, and hearts from WT mice were used as positive controls (lane 1). For WT samples, the total protein loaded was $1 / 10(15 \mu \mathrm{g})$ of the quantity in the other lanes $(150 \mu \mathrm{g})$. Quantification performed by densitometric analysis of autoradiographic bands followed by normalization with the quantity of total protein loaded onto the gel showed a dystrophin recovery of $8 \pm 0.6 \%$ in quadriceps and $7 \pm 0.17 \%$ in diaphragm $(P=0.0002)$ of $\mathrm{ZM} 2-\mathrm{AON}$-treated mice with respect to WT control mice. The results are representative of three separate experiments. Data are given as means \pm S.E.M. and the statistical significance has been calculated with the Student's $t$-test for unpaired data.

and exon-23 skipping are still measurable in skeletal muscles (quadriceps and diaphragms) of ZM2-AON treated $\mathrm{mdx}$ mice, as highlighted by immunofluorescence, western blot and ESRA analysis.

Previous works on the mdx mouse have suggested that levels of $30 \%$ of dystrophin or more are protective for mice $[38,39]$, and Neri et al. demonstrated that dystrophin mRNA and protein levels between $29 \%$ and $57 \%$ of control muscle are sufficient to avert muscular dystrophy in humans, when the protein is uniformly present in all muscle fibres [40]. Though we are well aware that the rescue percentages detected in our experiments are much lower, we do believe that these results could be a starting point for the optimization of a treatment featuring ZM2-AON complexes. In the future, we will work to clarify both the kinetic and dynamic process underlying NP-AON treatments by studying the biodistribution/clearance of ZM2-AON complexes and optimizing their dose regimen. Work will also be necessary to fine-tune their kinetics and devise novel formulations, taking advantage of the intrinsic physical plasticity of ZM2 nanoparticles. Indeed the protective effect exerted by NPs on AONs, which undoubtedly plays a role in persistence of the protein by means of prolonging the beneficial effect of AON treatment certainly encourages the exploration of innovative administration routes of this compound.

\section{Acknowledgments}

The Telethon Italy Grant GGP09093 (to A. Ferlini, Department of Experimental and Diagnostic Medicine, Section of Medical Genetics, University of Ferrara, Ferrara, Italy) is acknowledged. Thanks also are due to the TREAT-NMD Network of Excellence of EU FP7 no. 036825 (to partner FTELE-Italy) for work carried out within WP8.2. (A. Ferlini, member).

\section{References}

[1] A. Aartsma-Rus, J. C. T. Van Deutekom, I. F. Fokkema, G. J. B. Van Ommen, and J. T. Den Dunnen, "Entries in the Leiden Duchenne muscular dystrophy mutation database: an overview of mutation types and paradoxical cases that confirm the reading-frame rule," Muscle and Nerve, vol. 34, no. 2, pp. 135-144, 2006.

[2] A. Aartsma-Rus and G. J. B. Van Ommen, "Antisensemediated exon skipping: a versatile tool with therapeutic and research applications," RNA, vol. 13, no. 10, pp. 1609-1624, 2007.

[3] E. P. Hoffman, "Skipping toward personalized molecular medicine," The New England Journal of Medicine, vol. 357, no. 26, pp. 2719-2722, 2007.

[4] L. Du and R. A. Gatti, "Progress toward therapy with antisense-mediated splicing modulation," Current Opinion in Molecular Therapeutics, vol. 11, pp. 116-123, 2009.

[5] J. C. van Deutekom, A. A. Janson, I. B. Ginjaar et al., "Local dystrophin restoration with antisense oligonucleotide PRO051," The New England Journal of Medicine, vol. 357, no. 26, pp. 2677-2686, 2007.

[6] M. Kinali, V. Arechavala-Gomeza, L. Feng et al., "Local restoration of dystrophin expression with the morpholino oligomer AVI-4658 in Duchenne muscular dystrophy: a single-blind, placebo-controlled, dose-escalation, proof-ofconcept study," The Lancet Neurology, vol. 8, no. 10, pp. 918928, 2009.

[7] N. M. Goemans, M. Tulinius, J. T. van den Akker et al., "Systemic administration of PRO051 in Duchenne's muscular dystrophy," The New England Journal of Medicine, vol. 364, no. 16 , pp. 1513-1522, 2011.

[8] S. Cirak, V. Arechavala-Gomeza, M. Guglieri et al., "Exon skipping and dystrophin restoration in patients with Duchenne muscular dystrophy after systemic phosphorodiamidate morpholino oligomer treatment: an open-label, phase 2, doseescalation study," The Lancet, vol. 378, no. 9791, pp. 595-605, 2011.

[9] J. H. Williams, R. C. Schray, S. R. Sirsi, and G. J. Lutz, "Nanopolymers improve delivery of exon skipping oligonucleotides and concomitant dystrophin expression in skeletal muscle of mdx mice," BMC Biotechnology, vol. 8, article 35, 2008.

[10] Y. Kim, M. Tewari, J. D. Pajerowski et al., "Polymersome delivery of siRNA and antisense oligonucleotides," Journal of Controlled Release, vol. 134, no. 2, pp. 132-140, 2009.

[11] S. R. Sirsi, R. C. Schray, X. Guan et al., "Functionalized PEGPEI copolymers complexed to exon-skipping oligonucleotides improve dystrophin expression in mdx mice," Human Gene Therapy, vol. 19, no. 8, pp. 795-806, 2008.

[12] W. Tao, J. P. Davide, M. Cai et al., "Noninvasive imaging of lipid nanoparticle-mediated systemic delivery of smallinterfering RNA to the liver," Molecular Therapy, vol. 18, no. 9, pp. 1657-1666, 2010. 
[13] X. Z. Yang, S. Dou, T. M. Sun, C. Q. Mao, H. X. Wang, and J. Wang, "Systemic delivery of siRNA with cationic lipid assisted PEG-PLA nanoparticles for cancer therapy," Journal of Controlled Release, vol. 156, pp. 203-211, 2011.

[14] H. de Martimprey, J. R. Bertrand, C. Malvy, P. Couvreur, and C. Vauthier, "New core-shell nanoparticules for the intravenous delivery of sirna to experimental thyroid papillary carcinoma," Pharmaceutical Research, vol. 27, no. 3, pp. 498$509,2010$.

[15] I. Bertholon, C. Vauthier, and D. Labarre, "Complement activation by core-shell poly(isobutylcyanoacrylate)- polysaccharide nanoparticles: influences of surface morphology, length, and type of polysaccharide," Pharmaceutical Research, vol. 23, no. 6, pp. 1313-1323, 2006.

[16] M. E. Davis, J. E. Zuckerman, C. H. J. Choi et al., "Evidence of RNAi in humans from systemically administered siRNA via targeted nanoparticles," Nature, vol. 464, no. 7291, pp. 10671070, 2010.

[17] P. Rimessi, P. Sabatelli, M. Fabris et al., "Cationic PMMA nanoparticles bind and deliver antisense oligoribonucleotides allowing restoration of dystrophin expression in the $\mathrm{mdx}$ mouse," Molecular Therapy, vol. 17, no. 5, pp. 820-827, 2009.

[18] A. Ferlini, P. Sabatelli, M. Fabris et al., "Dystrophin restoration in skeletal, heart and skin arrector pili smooth muscle of $\mathrm{mdx}$ mice by ZM2 NP-AON complexes," Gene Therapy, vol. 17, no. 3, pp. 432-438, 2010.

[19] H. Heemskerk, C. de Winter, P. Van Kuik et al., "Preclinical PK and PD studies on 2'-O-methyl-phosphorothioate RNA antisense oligonucleotides in the mdx mouse model," Molecular Therapy, vol. 18, no. 6, pp. 1210-1217, 2010.

[20] A. Ferlini and P. Rimessi, "Exon skipping quantification by real-time PCR," Methods in Molecular Biology, vol. 867, pp. 189-199, 2012.

[21] P. Spitali, P. Rimessi, M. Fabris et al., "Exon skipping-mediated dystrophin reading frame restoration for small mutations," Human Mutation, vol. 30, no. 11, pp. 1527-1534, 2009.

[22] C. A. Sewry, "Immunocytochemical analysis of human muscular dystrophy," Microscopy Research and Technique, vol. 48, no. 3-4, pp. 142-154, 2000.

[23] R. Kayali, F. Bury, M. Ballard, and C. Bertoni, "Site-directed gene repair of the dystrophin gene mediated by PNAssODNs," Human Molecular Genetics, vol. 19, no. 16, pp. 32663281, 2010.

[24] S. Fletcher, K. Honeyman, A. M. Fall, P. L. Harding, R. D. Johnsen, and S. D. Wilton, "Dystrophin expression in the mdx mouse after localised and systemic administration of a morpholino antisense oligunucleotide," Journal of Gene Medicine, vol. 8, no. 2, pp. 207-216, 2006.

[25] J. Alter, F. Lou, A. Rabinowitz et al., "Systemic delivery of morpholino oligonucleotide restores dystrophin expression bodywide and improves dystrophic pathology," Nature Medicine, vol. 12, no. 2, pp. 175-177, 2006.

[26] B. Wu, H. M. Moulton, P. L. Iversen et al., "Effective rescue of dystrophin improves cardiac function in dystrophin-deficient mice by a modified morpholino oligomer," Proceedings of the National Academy of Sciences of the United States of America, vol. 105, no. 39, pp. 14814-14819, 2008.

[27] N. Jearawiriyapaisarn, H. M. Moulton, P. Sazani, R. Kole, and M. S. Willis, "Long-term improvement in mdx cardiomyopathy after therapy with peptide-conjugated morpholino oligomers," Cardiovascular Research, vol. 85, no. 3, pp. 444453, 2010.

[28] H. Yin, A. F. Saleh, C. Betts et al., "Pip5 transduction peptides direct high efficiency oligonucleotide-mediated dystrophin exon skipping in heart and phenotypic correction in $\mathrm{mdx}$ mice," Molecular Therapy, vol. 19, no. 7, pp. 1295-1303, 2011.

[29] H. A. Heemskerk, C. L. de Winter, S. J. de Kimpe et al., "In vivo comparison of 2'-O-methyl phosphorothioate and morpholino antisense oligonucleotides for Duchenne muscular dystrophy exon skipping," Journal of Gene Medicine, vol. 11, no. 3, pp. 257-266, 2009.

[30] L. L. Qi, A. Rabinowitz, C. C. Yun et al., "Systemic delivery of antisense oligoribonucleotide restorers dystrophin expression in body-wide skeletal muscles," Proceedings of the National Academy of Sciences of the United States of America, vol. 102, no. 1, pp. 198-203, 2005.

[31] B. Wu, P. Lu, E. Benrashid et al., "Dose-dependent restoration of dystrophin expression in cardiac muscle of dystrophic mice by systemically delivered morpholino," Gene Therapy, vol. 17, no. 1, pp. 132-140, 2010.

[32] S. Fletcher, K. Honeyman, A. M. Fall et al., "Morpholino oligomer-mediated exon skipping averts the onset of dystrophic pathology in the mdx mouse," Molecular Therapy, vol. 15, no. 9, pp. 1587-1592, 2007.

[33] N. Jearawiriyapaisarn, H. M. Moulton, B. Buckley et al., "Sustained dystrophin expression induced by peptide-conjugated morpholino oligomers in the muscles of mdx mice," Molecular Therapy, vol. 16, no. 9, pp. 1624-1629, 2008.

[34] A. Malerba, F. C. Thorogood, G. Dickson, and I. R. Graham, "Dosing regimen has a significant impact on the efficiency of morpholino oligomer-induced exon skipping in mdx mice," Human Gene Therapy, vol. 20, no. 9, pp. 955-965, 2009.

[35] H. M. Moulton and J. D. Moulton, "Morpholinos and their peptide conjugates: therapeutic promise and challenge for Duchenne muscular dystrophy," Biochimica et Biophysica Acta, vol. 1798, no. 12, pp. 2296-2303, 2010.

[36] D. K. Monteith, M. J. Horner, N. A. Gillett et al., "Evaluation of the renal effects of an antisense phosphorothioate oligodeoxynucleotide in monkeys," Toxicologic Pathology, vol. 27, no. 3, pp. 307-317, 1999.

[37] E. S. Ferdinandi, A. Vassilakos, Y. Lee et al., "Preclinical toxicity and toxicokinetics of GTI-2040, a phosphorothioate oligonucleotide targeting ribonucleotide reductase R2," Cancer Chemotherapy and Pharmacology, vol. 68, no. 1, pp. 193205, 2011.

[38] D. J. Wells, K. E. Wells, E. A. Asante et al., "Expression of human full-length and minidystrophin in transgenic mdx mice: implications for gene therapy of Duchenne muscular dystrophy," Human Molecular Genetics, vol. 4, no. 8, pp. 12451250, 1995.

[39] S. F. Phelps, M. A. Hauser, N. M. Cole et al., "Expression of full-length and truncated dystrophin mini-genes in transgenic mdx mice," Human Molecular Genetics, vol. 4, no. 8, pp. 12511258, 1995.

[40] M. Neri, S. Torelli, S. Brown et al., "Dystrophin levels as low as $30 \%$ are sufficient to avoid muscular dystrophy in the human," Neuromuscular Disorders, vol. 17, no. 11-12, pp. 913918, 2007. 

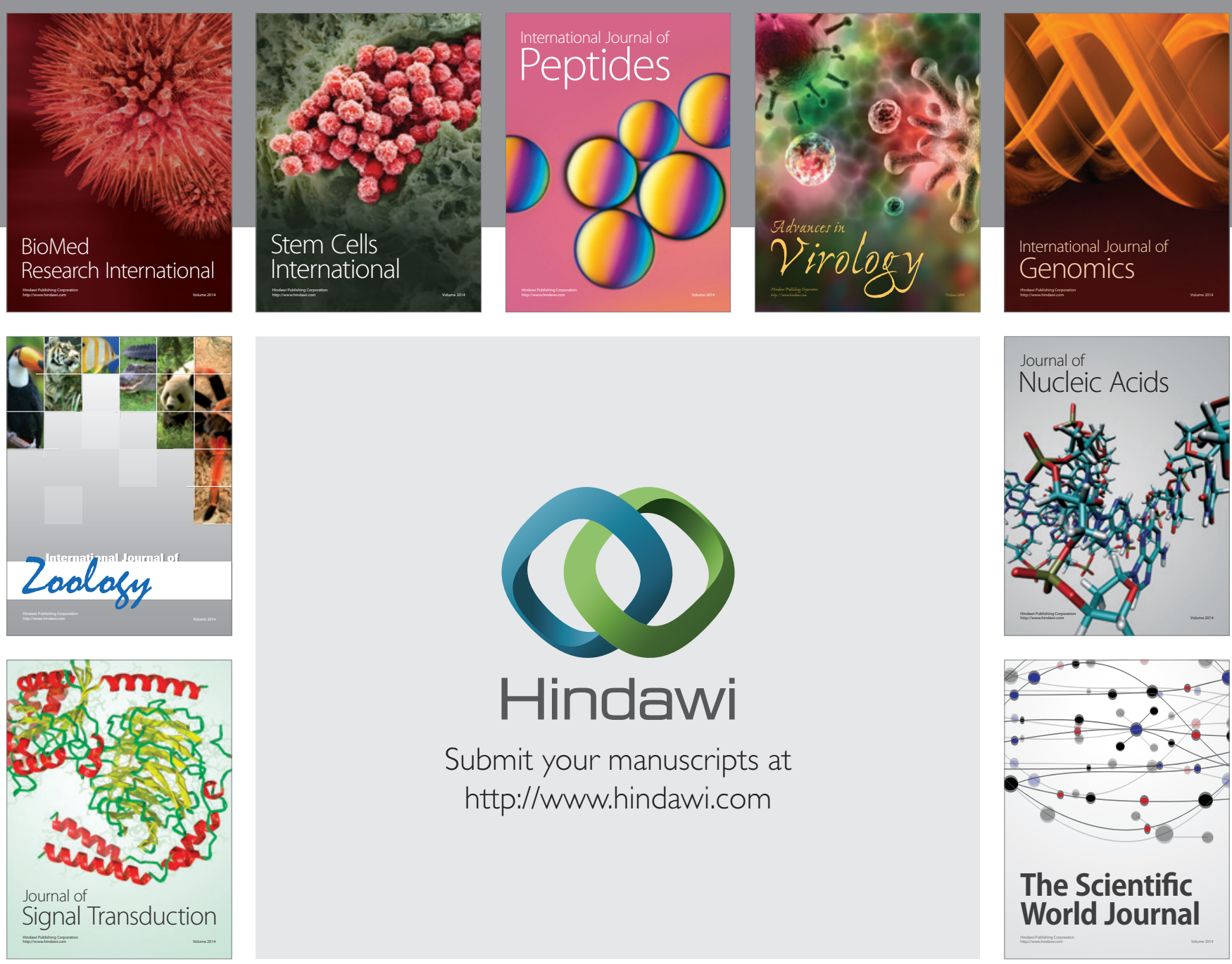

Submit your manuscripts at

http://www.hindawi.com
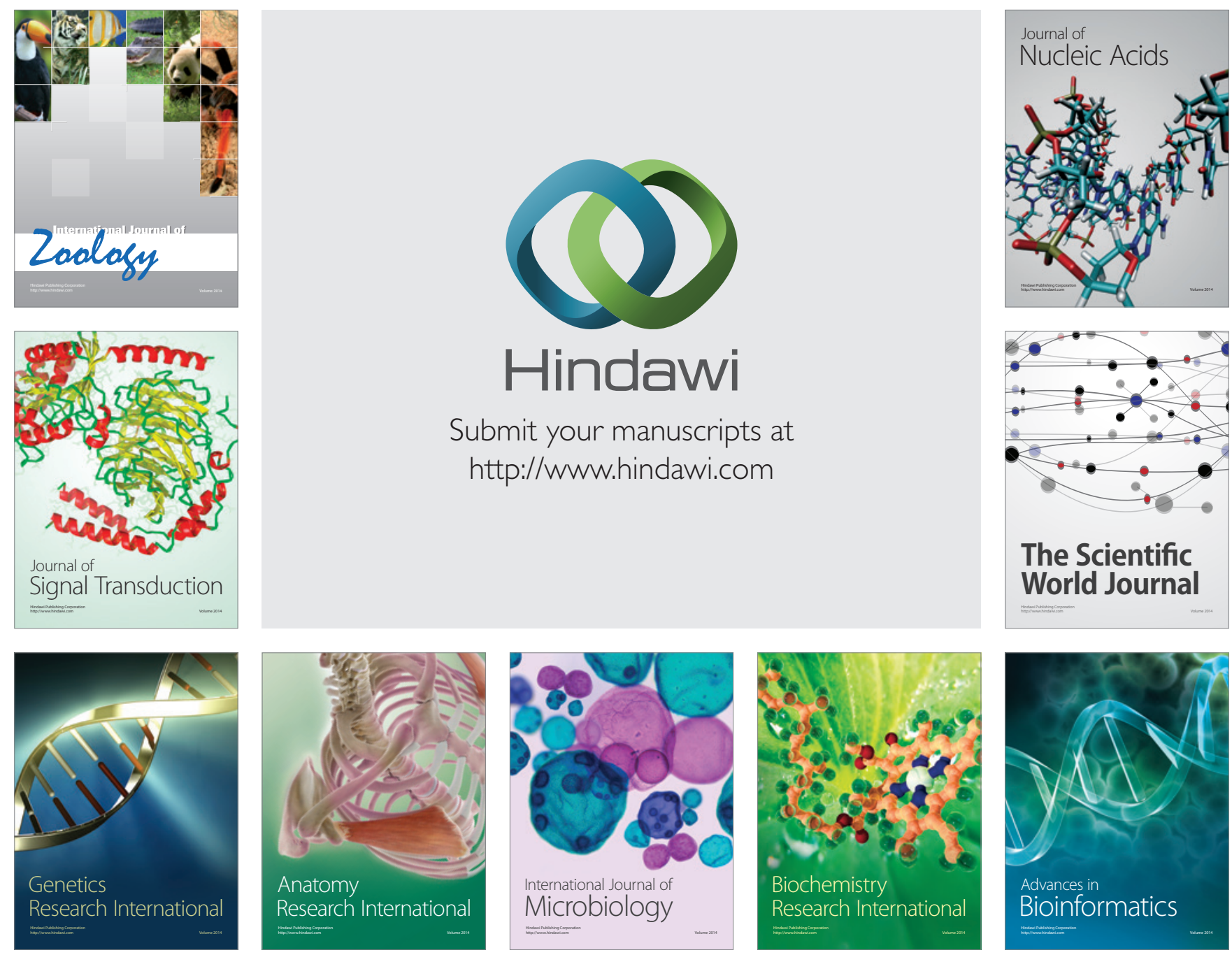

The Scientific World Journal
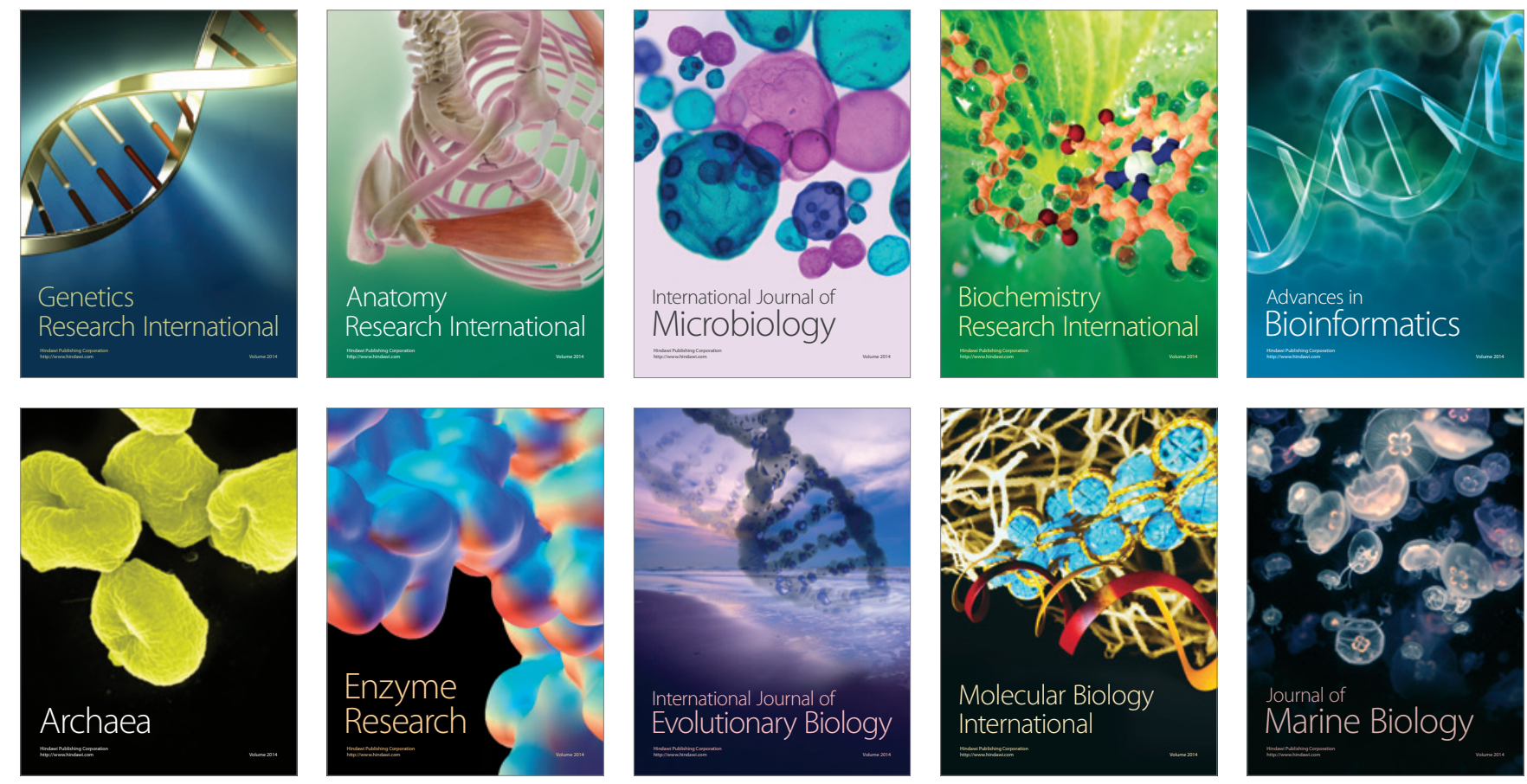